Der Autor Schwokowski hat das Verdienst, zum 150. Geburtstag dieses bedeutenden Leipziger Chirurgen ein äusserst informatives Symposium organisiert zu haben. Leipzig war vor dem Zweiten Weltkrieg neben Berlin das bedeutendste Zentrum der Medizin, wozu Trendelenburg wesentlich beigetragen hat. Das vorliegende Buch dokumentiert ebenfalls die Entwicklung des «medizinischen Viertels» der Messestadt. Interessant ist vor allem die Tatsache, dass 1906 in Leipzig unter Karl Sudhoff (1853-1938) das erste Institut für Geschichte der Medizin gegründet wurde.

Im zweiten Teil referieren namhafte Autoren aus ganz Deutschland über verschiedene chirurgische Teilgebiete, zu welchen Trendelenburg wesentlich beigetragen hatte.

Alles in allem kann das Buch als interessante historische Informationsquelle empfohlen werden.

Andreas P. Naef

Aristoteles. Werke in deutscher Übersetzung: Band 14: Parva naturalia. Teil 3: De insomniis, De divinatione per somnum. Übers. und erläutert von Philip J. van der Eijk. Berlin, Akademie-Verlag, cop. 1994. 396 S. DM 138.-. ISBN 3-05-002398-8.

Was die Antike über den Traum gedacht hat, ist auch noch im 20. Jh. mehrfach Anstoss wissenschaftlicher Auseinandersetzung gewesen: Man denke etwa an Freud, Jung, Binswanger oder Foucault. Freilich ist es nicht ganz einfach, den Zugang zur antiken Auseinandersetzung mit dem Traum zu finden. Sie ist vielgestaltig, zu einem grossen Teil schlecht überliefert und allzuoft nur mit der Hilfe von Spezialisten verständlich. Am bekanntesten ist wohl das Traumdeutungsbuch des Artemidor aus der römischen Kaiserzeit (2. Jh. n. Chr.). Die einzige erhaltene theoretische und systematische Abhandlung über Traum und Traummantik verdanken wir Aristoteles.

Übersetzung und Kommentar van der Eijks fallen in eine Zeit, in der sowohl eine Reihe von neuen Arbeiten zur antiken Traumdeutung im allgemeinen (so u.a. eben P.C. Miller 1994 zur Spätantike) als auch bei Aristoteles im besonderen zu verzeichnen sind. Im Rahmen der bisherigen Forschung (zuletzt D. Gallop, 1990, engl. Übers., Komm.) bedeuten sie einen wesentlichen Fortschritt und sind für das Verständnis der aristotelischen Traumtheorie sowie auch zentraler psychologischer Begriffe unerlässlich. Wie es in der Reihe «Aristoteles Werke in deutscher Übersetzung» üblich ist, fehlt der griechische Text. Dieser findet sich in der Textausgabe von P. Siwek (1963, mit lateinischer Übersetzung und Kommentar), welche der älteren, aber vielfach noch immer als Grundlage benützten Arbeit von W. D. Ross eindeutig vorzuziehen ist.

Van der Eijk untersucht Zielsetzung, Methode und Struktur der beiden Schriften und ordnet diese in den Zusammenhang des aristotelischen Gesamtwerkes sowie ansatzweise auch der antiken Traumdeutung ein. Vor allem geht es ihm darum, die aristotelischen Gedankengänge zu erhellen, zunächst in der Einleitung (knapp 100 Seiten inklusive 30 Seiten Bibliographie mit Hinweisen auch zu den grundsätzlicheren geistesgeschichtlichen Themen, leider aber ohne das wichtige Buch von J. Amat, 1985, zu nennen), dann auch in den gut 200 Seiten klaren kommentierenden Anmerkungen, welche jeweils mit ausführlichen Paraphrasen des Textes beginnen. Die Zielsetzung des Autors und der gewählte Aufbau führen notwendigerweise zu einigen Überschneidungen und Wiederholungen. Die Übersetzung für sich allein genommen ist nicht überall ganz leicht verständlich und letztlich nur hilfreich in Zusammenhang 
mit den Erläuterungen. Sehr nützlich ist die Liste mit den Übersetzungen der wichtigsten griechischen Begriffe.

Aristoteles stand den Möglichkeiten der Traumdeutung anders als wohl die meisten seiner Zeitgenossen und paradoxerweise auch als manche seiner Rezipienten äusserst skeptisch gegenüber - überspitzt: Je hohler der Kopf, desto mantischer die Träume. Ähnlich wie die entsprechenden Schriften im hippokratischen und galenischen Korpus gestand Aristoteles indes Träumen eine diagnostische Funktion in der Medizin zu. Im Unterschied aber zur medizinischen Traumdeutung und mehr noch zu den zahlreichen an der Traumbedeutung interessierten antiken Theorien, welche Träume hinsichtlich ihres mantischen Gehalts und damit ihrer Verwendbarkeit klassifizieren, stossen wir bei Aristoteles auf eine nicht anwendungsorientierte Behandlung des Phänomens. Die Leistung des Aristoteles liegt - trotz einiger Unklarheiten - auf methodischem und begrifflichem Gebiet. Nachdem für das Verständnis der aristotelischen Auffassungen nun doch erhebliche Fortschritte erzielt worden sind, wäre es wichtig, die Rezeption der Traumtheorie des Aristoteles besser zu erforschen.

Beat Näf

\section{Companion encyclopedia of the history and philosophy of the mathematical scien-} ces. Ed. by Ivor Grattan-Guinness. London [etc.], Routledge, 1994. 2 vols. $£ ~ 150 .-$. ISBN 0-415-03785-9 (set).

Dieses zweibändige Werk beeindruckt durch seinen Umfang (1806 Seiten), durch seinen reichen Inhalt, den zweckmässigen Aufbau und die grosse Zahl kompetenter Mitarbeiter aus 18 verschiedenen Nationen. - Geschichte und Philosophie der mathematischen Wissenschaften sind nicht auf Stichworte verteilt, wie man dies vielleicht von einer Enzyklopädie zunächst erwarten würde, sondern nach sachlichen Gesichtspunkten gegliedert: Nach einer Einleitung folgt ein erster Abschnitt «Ancient and non-Western tradition» mit kurzen Darstellungen nicht nur der babylonischen, ägyptischen, griechischen, byzantinischen Mathematik und der Mathematik in der islamischen Kultur, sondern auch der Mathematik in zahlreichen andern Ländern und Kulturen. Schon die Lektüre dieses Abschnitts zeigt die Reichhaltigkeit und die Weite, die der Herausgeber und seine Mitarbeiter angestrebt und auch realisiert haben. Der nächste Abschnitt «The Western Middle Ages and the Renaissance» bringt neben jenen Themen, die hier sowieso erwartet werden (z.B. Euklid- und Archimedes-Rezeption, Coss, Logarithmen usw.) auch willkommene Ausführungen zur Mechanik und zur Optik, zur Theorie der musikalischen Intervalle und schliesslich zum philosophischen Kontext der mittelalterlichen Mathematik und jener der Renaissance. Die nächsten acht Teile stellen dann die Entwicklung der Mathematik (einschliesslich der mathematischen Physik) seit dem frühen 17. Jahrhundert bis heute dar, jetzt aber aufgegliedert nach den Zweigen, in die sich die Mathematik mit der Zeit gegliedert hat. Auch diese Teile überraschen den Leser jedesmal wieder durch ihren Inhaltsreichtum. So findet man zum Beispiel im Abschnitt «Probability and statistics, and the social sciences» - nebst vielen anderen - kurzgefasste Darstellungen über die Geschichte der Kombinatorik, über die Frühgeschichte der mathematischen Wahrscheinlichkeit, die Entwicklung der Versicherungsmathematik, der Fehlertheorie, der Theorie des Schätzens und Testens, der Anwendungen der Wahrscheinlichkeitsrechnung und der mathematischen Statistik in den verschiedensten Gebieten, weiter aber 